\title{
Research and application of medical management information technology in tobacco enterprise
}

\author{
Jin Xu, Jinxing Zhang, Wei Cheng, Jiulian Luo, Hui Lou, Min Zhang \\ China Tobacco Zhejiang Industrial Co. LTD., Hangzhou, China
}

Keywords: Employee health, health care, information technology

\begin{abstract}
Through the study of the disadvantages of the infirmary and the domestic and foreign related development level, this research proposes the technical analysis program of the medical system, and establishes the information and data management and control mechanism. Through information management, the final implementation of the fine management of employee health management and control, improve efficiency and scientific management.
\end{abstract}

\section{Introduction}

Infirmary bear to protect the health of the staff, including the diagnosis and treatment of common diseases and referral services, the management of drugs (including procurement, storage, use, disposal), employee medical expenses reimbursement management. At present, the tobacco enterprise internal infirmary various management work is still relatively traditional, all kinds of information (including prescription drugs, medical expenses, and employees etc.) mostly used paper documents to record, archive, information search is very difficult.

When paper is used as a carrier, this method can greatly affect the speed of information search, and save time is limited. Secondly, all kinds of information is relatively independent of each other, lack of internal links, cannot provide the basis for the subsequent analysis of the data, it cannot provide guidance for the improvement of enterprise management. Third, low-level management of information to bring the extensive management of the medical work.

\section{The research situations at home and aboard}

Medical institutions in the development of information technology abroad earlier, the basic level of information technology services, networking, data is also building a more perfect. Family doctor, emergency center, general hospital and patients, insurance companies and other medical information sharing more successful. National Health Service system in the United Kingdom (NHS). The establishment of a reasonable classification model of diagnosis and treatment not only needs the understanding and cooperation between the two sides, but also requires a high level of medical information sharing.

With the great leap forward development of economy and the significant adjustment of economic structure, domestic health industry is developing rapidly. Integrated hospital information construction is more perfect, the grass-roots hospital is the construction according to the local financial strength, regional differences, but the grass-roots medical institutions and medical information sharing between hospitals is construction slow. Health care sharing between provinces 
and municipalities are also building more slowly. While the enterprise internal medical institutions of basic medical services, with a variety of state owned enterprises bankruptcy reorganization, a welfare nature of grassroots medical institutions to shrink more powerful, not only reflected in the number, is also reflected in the quality and quantity of the former agencies and staff have been reduced, the latter is mainly reflected in the quality of service and to provide the medical service.

\section{Internal medical system technical analysis program}

The research and application of information management and control in the infirmary is extremely urgent. This needs to change the current mode of information acquisition, and then establishes a complete and perfect information network analysis data analysis model, in order to truly improve Infirmary management level, save medical resources for the enterprise, for employees to save the medical cost, and improve Infirmary worker health and ability for enterprise production safety play a security role practical.

It consists of three stages. In the first stage: the existing business of the infirmary is sorted out, and the relationship among the various operations is sorted out, and the business and its correlation degree model is established. The second stage: the establishment of a set of information collection system to collect health information, including but not limited to the prescription, medical records, medical expenses, etc. The third stage: the establishment of data analysis system and control mechanism, to continue to track the management of employee health, fine management of drug procurement, medical expenses, etc.

First stage: In order to sort out the existing business of the infirmary, and to sort out the relationship between the business, the establishment of business and its correlation degree model. The main work is to clarify the Infirmary in the business and employee health, including the outpatient records, prescription records, Drug Administration (including demand, procurement, inventory, warehousing, scrap, etc.) the management of medical devices, medical alcohol supplies management, and with the staff of the archives management, employee health employees of major diseases of archives management, employee records management, chronic disease management, employee reimbursement of medical expenses of medical insurance of employees and their families, there is Infirmary aspects, such as job titles, annual medical institutions appraisal management etc.. And the degree of health information related to the business and the information construction of the medical staff to establish the degree of relevance model to assess the importance of each business and frequency.

In the implementation process, the information management mechanism on the one hand may refer to the basic hospital, at the same time to visit and study the Infirmary brothers enterprises, understand the informatization construction of grassroots medical institutions, at the same time to find information about the construction of grass-roots medical institutions of the literature at home and abroad to enhance the level of service.

The second stage: According to the working process of the medical office, the establishment of a set of information collection system to collect health information, including but not limited to the prescription, medical records, medical expenses, etc.. The specific work is based on the first stage, the establishment of an information collection system, the software system can timely record Infirmary emergency information, prescription information, drug management information, and docking of medical expenses, employee medical archives, and tracking maanagement of key information, key staff, worker health and safety. In the software system to complete the design phase, with the characteristics of the enterprises, to meet the demands of daily management Infirmary also set aside to upgrade extra functions of space, focus on solving the key technology and the needs of the project involved. At the same time in the process of system development, 
debugging, continuous business integration, to solve the problem of data records, save, analysis, finishing, and finally improve the implementation of software systems development. On the basis of this, the medical management module is designed. As shown in Figure 1.

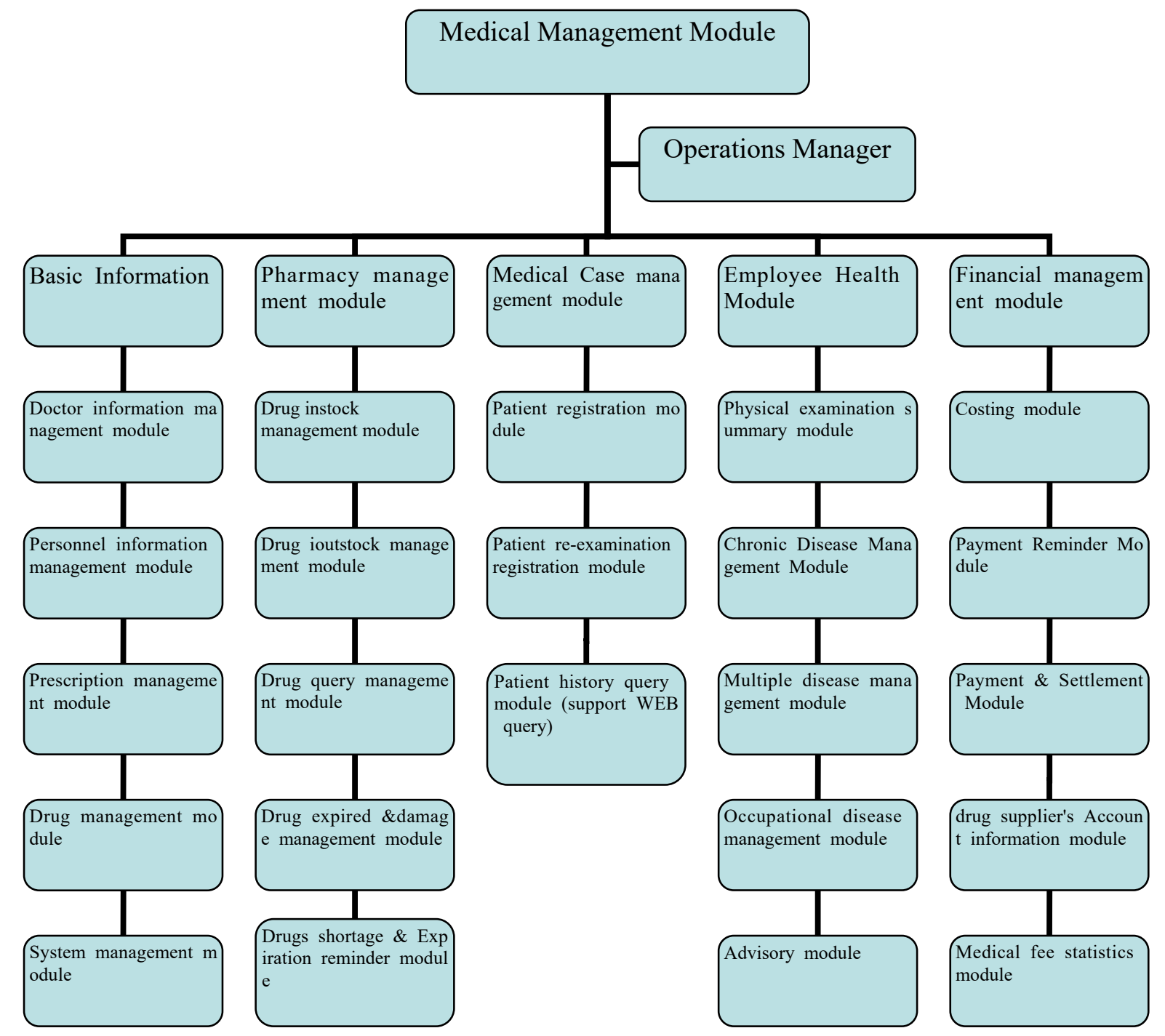

Figure 1 Medical management function module

The third stage: The establishment of data analysis system and control mechanism. According to the Infirmary information management system is established, according to the data on prescription management, drug management and information Infirmary overall analysis and improvement, to complete the establishment of the management mechanism, improve the Infirmary management level, and can provide the research results, provide support for enterprise decision-making.

\section{Establishment of medical system in tobacco enterprises}

According to the Infirmary medical information system, as shown in figure2, the collection and the contents of health staff, including major emergency records, prescription records, Drug Administration (including demand, procurement, inventory, warehousing, scrap, etc.) the management of medical devices, medical alcohol consumption material management, and with the relevant staff, employee health records management, staff file management, staff of major diseases, chronic disease archives management, staff management, reimbursement of medical expenses of 
health care and other family members of staff. At the same time according to the information collected, the data collation and analysis of comprehensive management and control work, to fully understand the health status of the staff, and fine control of management and operation of enterprises of drug procurement, such as the use of medical expenses.

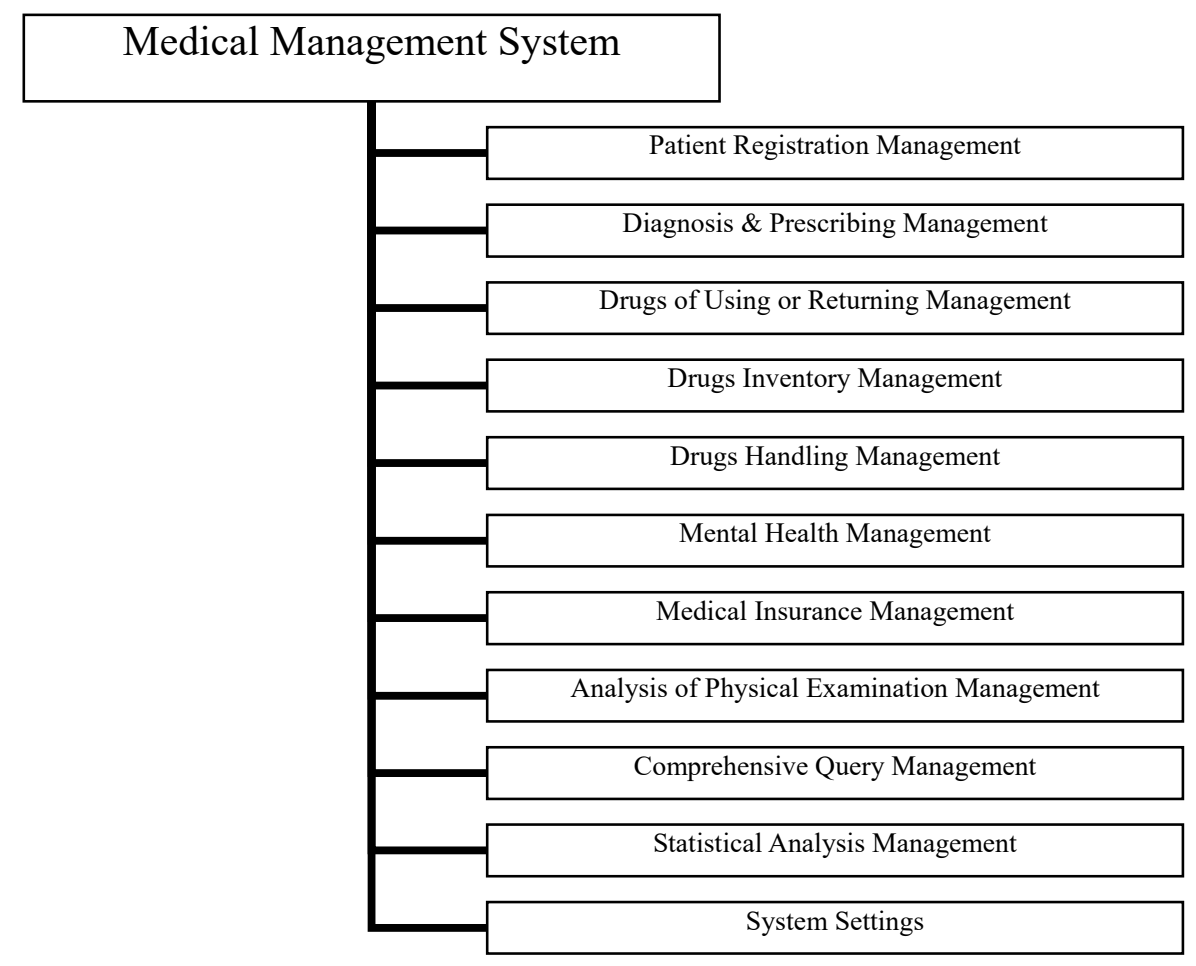

Figure 2 Medical Management System

Registration management: According to the medical management system of the existing staff to Infirmary treatment activities are required to work permit registration departments, trade unions and other activities or medicine need medicine, required by the department approval of the leadership, the use of public account registration management.

The diagnosis of prescription management: according to the Ministry of Health issued the "prescription management method", Infirmary doctors according to the patient's condition, the actual need for disease diagnosis, prescription, prescription is generally not more than 7 days the amount for some chronic diseases or special circumstances (such as long-term assignments etc.), prescription dosage can be appropriately extended. The doctor in the diagnosis of disease, prescription, should be careful to ask the patient's condition, medication taboo, allergic history, after the kitchen, should tell the drug use methods and side effects, etc..

Lead the management of drug withdrawal: pharmacy pharmacist according to the doctor's prescription, carefully check the amount of medication is reasonable, whether appropriate, according to the FIFO principle, according to the distribution of drugs in prescription, drug use, and told the storage method and special circumstances; for the unreasonable prescription should be returned, please ask the doctor to prescribe.

Drug storage management: pharmacists according to drug reception, timely grasp of the pharmacy intake, intake, sunrise and inventory, do the monthly drug account. According to the monthly amount of pharmacists entry, procurement plans, report Infirmary leading audit, approved timely procurement of appropriate drugs, drug inventory management according to the type size, storage method, validity period etc.

Drug treatment: the pharmacist management according to the drug and inventory of effective 
management for drug consumption, should communicate with the doctor, consider whether there is abuse for drug shortage, impersonator phenomenon; should remind doctors to limit the use and timely purchase of similar drugs; drug should remind the doctor for the future, timely treatment, adjust the purchase volume at the same time. Reduce inventory; for expired, damaged the drug, should be in accordance with the provisions of timely disposal and write off inventory at the same time, the lessons learned, appear to reduce such phenomenon.

Mental health management: Infirmary doctors according to the relevant mental health examination for employees in the detection of data, establishment of employee psychological archives, also have mental illness depression, bipolar disorder and so on with employees to focus on monitoring, timely grasp the dynamic mental health of employees.

The management of medical insurance for employees: Infirmary doctor generated during the treatment of medical expenses, reimbursement audit according to the company's pay system, at the same time for the administration of registration of affiliated relatives.

Medical management analysis: Infirmary for employee health examination, physical examination occupation disease registration and management, all the staff of the regular analysis of the physical examination rate, disease, occupation disease, and chronic disease management, major diseases.

Comprehensive query management: Infirmary for employees basic information (such as age, gender, Department, contact), health status, medical expenses, medical insurance use, chronic disease, occupation disease and other data query, maintenance and management.

Statistical analysis: Infirmary management for the collected data, the staff health insurance, drug use, Infirmary entry and other medical information collection, collation, statistics and analysis of effective management.

System settings: basic configuration management of the medical management system of Zhejiang Hangzhou cigarette factory, including but not limited to increase module management functions, enhanced data statistics, data with other factory, improve the use of relative access permissions to access the system.

The management and control mechanism based on the analysis of information data is established, which is used to control and manage the drug purchasing, the use of medical expenses, health tracking and so on.

\section{Problems and deficiencies in the implementation of the project}

Information system software is more dependent on desktop computers and enterprise internal network, and in the specific use of the process, there have been many problems such as network failures led to the use of the system cannot be landed.

At the same time in the development of smart phones today, the future development and improvement direction will be the development of the software system can be applied at the same time in the smart phone, so the acquisition and update of information will be more efficient.

\section{References}

[1] Chen Jinxiong, Wang Hailin. Towards intelligent medical treatment: reconstruction of digital hospital theory system [M], 2014:101-150

[2] Zhang Ying. 10 cases of hospital management consulting (2001 - 2011) [M], 2011:320-415

[3] Li Xiaohua. Hospital information technology and application [M], 2014:241-380

[4] Cao Jianwen. Modern hospital management [M], 2003:28-44

[5] Zhang Lulu, Li Jing, Xu Zuming. Advanced hospital management [M], 2004 :118-123 